\title{
Sanitation and Analysis of Operation Data in Energy Systems
}

\author{
Gerhard Zucker ${ }^{1}$, Usman Habib ${ }^{1, *}$, Max Blöchle ${ }^{1}$, Florian Judex ${ }^{1}$ and Thomas Leber ${ }^{2}$ \\ Received: 3 September 2015 ; Accepted: 3 November 2015 ; Published: 11 November 2015 \\ Academic Editor: Chi-Ming Lai \\ 1 AIT Austrian Institute of Technology, Giefinggasse 2, Vienna 1210, Austria; \\ gerhard.zucker@ait.ac.at (G.Z.); max.bloechle@ait.ac.at (M.B.); florian.judex@ait.ac.at (F.J.) \\ 2 Omnetric GmbH, Ruthnergasse 3, Vienna 1210, Austria; thomas.leber@omnetric.com \\ * Correspondence: usman.habib@ait.ac.at; Tel.: +43-660-3442231
}

\begin{abstract}
We present a workflow for data sanitation and analysis of operation data with the goal of increasing energy efficiency and reliability in the operation of building-related energy systems. The workflow makes use of machine learning algorithms and innovative visualizations. The environment, in which monitoring data for energy systems are created, requires low configuration effort for data analysis. Therefore the focus lies on methods that operate automatically and require little or no configuration. As a result a generic workflow is created that is applicable to various energy-related time series data; it starts with data accessibility, followed by automated detection of duty cycles where applicable. The detection of outliers in the data and the sanitation of gaps ensure that the data quality is sufficient for an analysis by domain experts, in our case the analysis of system energy efficiency. To prove the feasibility of the approach, the sanitation and analysis workflow is implemented and applied to the recorded data of a solar driven adsorption chiller.
\end{abstract}

Keywords: data sanitation workflow; machine learning; $k$-means clustering; outlier detection; z-score normalization; adsorption chillers; first principle

\section{Introduction}

Data acquisition in buildings and building-related energy systems is primarily implemented for control purposes and for subsequent manual examination of faulty operations. There are different systems available for managing buildings such as XAMControl [1] by Evon, Enterprise building integrator (EBI) [2] by Honeywell and Desigo CC [3] by Siemens, which offer supervisory control systems with additional features like historic data storage and data analysis. However, practical experience with historical monitoring data from sensors has shown that it is often inaccurate and incomplete [4-6]. There are issues like changes in configuration that are not tracked correctly, bad calibration of sensors, outliers in the data, or issues in the data storage structure. Data sanitation is the process of correcting the monitoring data in order to increase data quality. This includes sanitation tests to check whether the data are physically plausible and in acceptable process range as well as reconstruction of missing or implausible data. It has been shown in [7] that conventional analysis using the calculations found in norms and standards, e.g., EN 15316 yields misleading results if data quality is low.

As the analysis of building efficiency is mostly done for large buildings or building stocks, the amount of data required to achieve meaningful results is typically heterogeneous. Analysis with respect to inefficiencies, faults and failures therefore is a time consuming and thus expensive task; however, it carries the potential for significant energy efficiency improvements during operation. One way to lessen this effort is to assist data sanitation and detection of inefficiencies through automated 
processes. By coupling machine learning algorithms and visualizations, the efforts for domain expert to analyze and interpret the data can be severely reduced. At the same time the configuration effort for setting up the sanitation process needs to be low.

During the use of such automated processes, it is prudent to preserve the original data: this allows one to determine the actual problems in the data which have been corrected. Furthermore, it may be required for auditing purposes. Finally, it also allows rerunning the sanitation process in case improvements in configuration have been identified.

The different methods presented in this paper are selected with regard to the effort it takes to analyze big amounts of data with a low availability of additional information, so called meta data. Also the amount of required domain knowledge (i.e., expertise on how the underlying processes work) shall be low; many of the steps described require little or no domain knowledge (as shown in Table 1).

Table 1. Overview of data analysis methods.

\begin{tabular}{ccc}
\hline Method/View & $\begin{array}{c}\text { Requires } \\
\text { Configuration }\end{array}$ & $\begin{array}{c}\text { Requires Domain } \\
\text { Knowledge }\end{array}$ \\
\hline Method: checking data access and availability & No & No \\
View: calendar view for data availability & No & No \\
Method: $k$-means clustering for duty cycle detection & No & No \\
Method: z-score and expectation maximization for outlier detection & No & No \\
Method: interpolation for gap sanitation & No & Yes ${ }^{1}$ \\
Method: setting process limits & Yes & Yes \\
Method: regression for sanitation of large gaps & No & Yes ${ }^{1}$ \\
View: histograms for on-state data only & No & Yes \\
Method: first principle (energy balance, COP) & Yes & No \\
View: legacy & No & No \\
View: snap-to-grid & No & No \\
\hline
\end{tabular}

${ }^{1}$ Requires basic information typically found on a data sheet.

The paper is organized in two main parts: in the first section the process of data sanitation and analysis is described together with the algorithms and visualizations that support the process. The second part (starting with Section 5) applies the process to a demonstration system with typical representative properties and data quality level.

For the scope of this paper the following naming conventions are used: a data point is a logical entity that represents a physical sensor value or state, which is usually a scalar (e.g., the data point T_LTre representing the temperature sensor measuring the return temperature in the Low-Temperature circuit). A data sample is the value that is taken at a certain point in time (e.g., T_LTre has $20^{\circ} \mathrm{C}$ at 10:00 o'clock). A data row is a set of data samples (e.g., all temperature values of T_LTre of yesterday). A batch of data is the block of data rows that is imported at once and to which the process described in this paper is applied to.

\section{State of the Art}

The goal of data sanitation is to improve data quality and thus increase confidence in the information that is gathered from the data. The properties of data quality are, amongst others, defined in [8]. In this paper, operational data of physical processes is used. The focus is therefore put on the following data quality properties: accessibility, credibility, completeness, consistent representation and interpretability.

Practical experience has shown that recorded operation data in building related energy systems needs to be validated before starting any detailed energy analysis. There are numerous methods available for data validation depending on the field in which they are used. The common methods are: indicator of status of sensor, physical range check, detection of gaps and constant values, 
tolerance band method, material redundancy detection, signal gradient test, extreme value check, physical and mathematical based models and various data mining techniques $[4,6,9]$.

The data can be labeled as $\mathrm{A}, \mathrm{B}$ and $\mathrm{C}$ on the basis of data validation techniques where A represents correct, $B$ represents doubtful and $C$ represents wrong data [9]. There is also a possibility of assigning a confidence value between 0 and 100 in order to provide refined information about data [10].

The detection of gaps is also an important factor to indicate the reliability of data; data with fewer gaps is considered better quality data. Experience shows that monitoring systems tend to fill missing data with the last available data sample, thus creating periods of constant data. Such hidden data gaps can be detected by doing an analysis of the variance, as sensors usually have some small variation of measurements with the exception of Boolean states [10].

Furthermore, it is recommended to use a time continuity check for the detection of unrealistic gradients. The time continuity check allows defining the maximum allowed difference between the measurements in a given interval of time [5].

In order to detect and diagnose faults in HVAC (heating, ventilation, air-conditioning) components, different diagnostic techniques are available: One can find faults by using some knowledge about the process, where the main focus is on first principles i.e., fundamental laws like energy balance or mass balance. On the other hand there are techniques that are based on the behavior of the system or process which is usually captured from historical data; Black box models are one of the examples of such techniques. The results from both these methods vary due to the properties of data and the fields in which they are used [11].

Data mining with respect to HVAC systems (heating, ventilation, air-conditioning) is currently mostly used with respect to optimization of system controls by adding data sources or forecasting capabilities. In Fan et al. [12], prediction models are developed for day ahead forecasts of building performance. In [13], the prediction model was used to detect occupancy to determine the right level of lighting, while in [14] principal component analysis was used to determine abnormal behavior in a single air handling unit. Schein et al. [15] on the other hand used a set of about 30 rules (mostly formulas based on the desired thermodynamic behavior of the unit) for the same purpose. On the other hand $\mathrm{Yu}$ et al. [16] used data mining to identify correlations in building energy consumption of HVAC. However, they but did not sufficiently verify whether the rules extracted and as a result the recommendations for changes in the system based on these are actually feasible. Zucker et al. [17] successfully used the X-Means algorithm to automate the detection of system states in order to examine operation data of adsorption chillers. Yu and Chan [18] also focused on chillers as a main component of energy consumption and use data envelopment to improve the energy management of chillers. Hong et al. [19] focused on retrofitting of high performance buildings and use data analytics for energy profiling and benchmarking.

\section{Sanitation and Analysis Workflow}

The requirements and constraints that led the design of this workflow are mainly application oriented. With regard to monitoring of energy systems the data is usually created as a side product of controlling a process, it is vendor and system specific with no established standardization of data formats, meta data or other high-level semantics. Figure 1 shows the workflow; the separate steps are elaborated in the following Sections 3.1-5. The workflow described here is based on the assumption that the steps shown in Figure 1 are executed on a batch of data that is imported in one bulk: if a set of data points for one month is imported, then the analysis is done on this one month of data, but does not affect data that has been imported earlier. The same applies for daily or annual import of data. For data that is imported continuously using an online machine-to-machine (M2M) interface (e.g., OPC Unified Architecture (UA), Representative State Transfer (REST) or Structured Query Language (SQL) based), the analysis period has to be set artificially to e.g., once per hour in order to keep the 
overhead low. Typically this is a process that is installed and fine-tuned by a human operator and once it is found to be trustworthy - scheduled to run automatically and periodically.

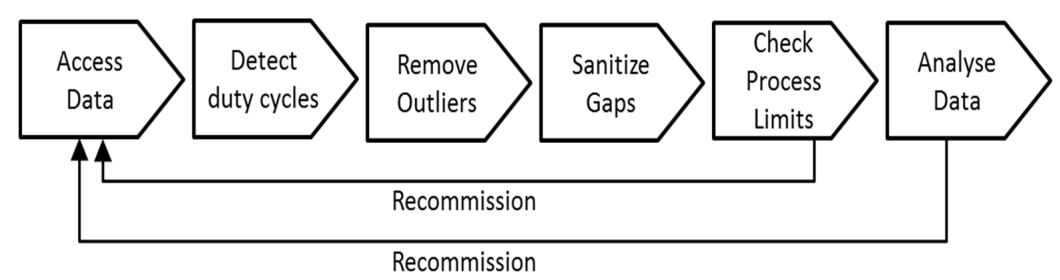

Figure 1. Data sanitation workflow.

When a new system is analyzed the recommended approach is to complete the whole workflow step-by-step and audit the single steps, i.e., closely observing whether the algorithms yield the expected results. Once a configuration (that is, a selection of required algorithms and their parameterization) is established and the algorithms are tuned until the result is satisfactory and the operator has confidence in the workflow, then the whole process is automated for all future batches of data.

In case the workflow reveals data quality issues in the "Check Process Limits" or "Analyse Data" step it is recommended to rerun all steps of the workflow and start from the raw data again, otherwise artifacts from previous sanitation steps may remain.

\subsection{Making Data Accessible}

The data being used for analysis usually originates from embedded controllers or management systems that have to ensure operation of the system, but are commonly not intended for data analysis. This results in different types of problems: sensors may operate correctly as far as operation is considered, but may not provide correctly calibrated values, thus resulting in incorrectly measured values. The recorded data is usually stored in a data container, which can be a database or just text files (for the sake of simplicity, it is referred as a container to describe any kind of data collection for monitoring data, including SQL databases as well as CSV files and proprietary data formats). The syntax of the data is not standardized; there is a plethora of proprietary data formats, both for the structure and for the values. This implies that the meta data, which is necessary to interpret the data rows in a container, is incomplete or ambiguous and needs processing before being able to proceed. Unless the container is strictly specified, including data formats for values, name spaces for data point naming, conventions on units, handling of missing data etc., there is manual effort required to make the data accessible. The solution that has been proven useful in practice is to create an import filter from the proprietary container format into an actual analysis system that has strict definitions on data storage. This way all following data analysis steps can operate on a unified system and be applied to data that stem from different systems.

\subsection{Duty Cycle Detection}

If the data originates from a system that has a duty cycle, meaning it switches between an On-state and an Off-state, it is advantageous to use this duty cycle for further analysis. The duty cycle period will be the base period for statistical analysis. In case there is no significant duty cycle, analysis is done on a batch of data, for example, the batch of data that is imported in one go. In case this is not feasible, e.g., because multiple years of data are imported at once, data has to be split into sub-batches with shorter periods, e.g., one week or one month. The definition of a period, may it be duty cycle or another period, is necessary in order to apply statistical analysis like outlier detection on data with small changes in boundary conditions.

If the system has a duty cycle, there may be a variable that indicates the current state of the machine, e.g., a binary signal from the controller. In this case the duty cycle detection is 
straight forward by using this variable. Otherwise the duty cycle has to be derived from other process variables such as temperature levels or mass flows. For this detection a robust algorithm is required that can automatically process the data batches. The $k$-means clustering algorithm has been successfully used for duty cycle detection [20,21]. $k$-means is a method of finding clusters in data based on the squared error criteria [22]. The $k$-means algorithm finds the k-partitions in such a way that in each partition the squared error between the mean $\left(\mu_{k}\right)$ of a cluster and the points in the cluster is minimized. Let $A=\left\{\mathrm{a}_{i}\right\}, i=1, \ldots, n$ be the set of $\mathrm{n}$ patterns and that is required to be clustered into a set of $K$ clusters, $C=\left\{C_{k}, k=1, \ldots, K\right\}$. Let $\mu_{k}$ be the mean of cluster $C_{k}$. The squared error between $\mu_{k}$ and the points in cluster $C_{k}$ is defined as:

$$
J\left(C_{k}\right)=\sum_{x_{i} \in C_{k}}^{n}\left\|x_{i}-\mu_{k}\right\|^{2}
$$

The goal of $k$-means is to minimize the sum of the squared error over all $K$ clusters:

$$
J(C)=\sum_{k=1}^{K} \sum_{x_{i} \in C_{k}}^{n}\left\|x_{i}-\mu_{k}\right\|^{2}
$$

The main steps of $k$-means algorithm are as follows:

1) Generate the initial partitions of $K$ clusters.

2) Assign the cluster to each pattern on the basis of its closest cluster center.

3) Calculate the new cluster centers.

4) Repeat Steps 2 and 3 until all the patterns are assigned the cluster.

The $k$-means algorithm detects the On/Off state of a system; a duty cycle is then defined to be the sequence of one On-state followed by one Off-state. The benefit of $k$-means is that the algorithm works on scalar values and does not need predefined process limits. The only configuration effort required is to tag the detected states as On-state or Off-state, for example, the high temperature cluster reflects the On-state, the low temperature cluster is the Off-state. The state and duty cycle detection is applied to the demo system in Section 5.3.

\subsection{Removal of Outliers}

Data that originates from the operation of a system typically contains samples that are not within the process limits (e.g., the allowed temperature range or the maximum possible mass flow in a process), but that do not necessarily indicate faulty operation of the system. In case the outliers are singletons or a limited amount of consecutive samples, it is reasonable to sanitize the data by removing the outliers and replacing them by physically plausible data samples. This task can be done by a human operator who requires only limited knowledge on the underlying physical processes (typically knowing the process limits is sufficient).

However, the challenge is to automate this process so that no human intervention is required. Algorithms from the domain of machine learning can be applied to solve this issue by clustering data and identifying the clusters which denote outliers. Clustering shall again be executed automatically with a minimum configuration effort, meaning that it shall be possible to detect outliers, even if information like process limits are missing.

The outlier detection process described here uses z-scores, see e.g., [23]. It operates automatically and does not require configuration. However, the performance of the outlier detection benefits from the duty cycle detection: z-score considers the mean and standard deviation of the whole data for normalization; this way outliers are better distinguishable from normal behavior, thus improving the clustering algorithm that identifies the outliers. 
Furthermore, systems with a duty cycle typically have different operation ranges during Onand Off-state. If the z-score would consider the whole duty cycle as one, there is a higher chance that it neglects or considers the correct data points as erroneous data points. To overcome this problem, this research suggests to derive the z-score separately for On-state and Off-state as following where $z$-score ${ }_{C y c l e}$ is the $z$-score for each state in a duty cycle, $X$ is the value of the sensor, $\mu_{\text {Cycle }}$ is the population mean of each state in the cycle, and $\sigma_{\text {Cycle }}$ is the standard deviation of each state in the cycle:

$$
\mathrm{z}-\text { score }_{\text {Cycle }}=\left(\mathrm{X}-\mu_{\text {Cycle }}\right) / \sigma_{\text {Cycle }}
$$

Expectation Maximization (EM) is a method for finding the maximum likelihood estimates of the data distribution when data is unknown or hidden. The EM clustering algorithm uses the finite Gaussian mixtures model, then tries to estimate a set of parameters until the anticipated convergence value is achieved. Now the mixture has $\mathrm{K}$ probability distributions in which each distribution represents one cluster. For each instance the class membership is assigned with the maximum probability. The working of EM algorithm can be defined as follows [24]:

1) Start with guessing the initial parameters: mean and standard deviation (in case the normal distribution model is being used).

2) Now enhance the parameters with $E$ (Expectation) and $M$ (Maximization) steps iteratively. In the E step, the membership probability for each instance on the bases of the initial parameters is calculated. Whereas in the M step the parameters are recalculated on the base of new membership likelihoods.

3) Allocate each instance of the data to the cluster with which it has the highest likelihood probability.

In order to enable the EM clustering algorithm to decide the number of clusters automatically, the cross validation method is used $[25,26]$. The cross validation is done using the following steps:

1) Initially the number of clusters is set to 1.

2) The training set is randomly divided into 10 folds.

3) The Expectation Maximization (EM) is applied to each fold using cross validation.

4) The average log likelihood of the 10 results is calculated; if the log likelihood is increased then the number of clusters is increased by 1 and it goes back to Step 2 again.

\subsection{Sanitation of Gaps}

Gaps in the recording of data occur in daily operation e.g., due to communication glitches or other interruptions in monitoring. Also the previously presented removal of outliers (Section 3.3) causes gaps in the data that need to be repaired. Two types of gaps can be distinguished: short gaps can be fixed by interpolation between the two adjacent samples. Sanitation of longer gaps requires a closer inspection of the data at hand and shall therefore be done in the analysis-step with support of a regression analysis as described in Section 5.6.1.

The definition of how long a gap shall be in order to be fixed by interpolation depends on the dynamics of the underlying process. For highly dynamic processes the gap period should be short, otherwise data quality decreases. A "hands-on" approach is to rely on the assumption that the monitoring system has a sample rate that is consistent with the dynamics of the system. Electric energy meters, for example typically take a sample every $15 \mathrm{~min}$, since this is adequate for observing the load profile. The same applies for slow thermal processes like heating and cooling in buildings. Under the assumption that the maximum relevant frequency in a process is correlated with the sample rate it is sensitive to define the measure the maximum length of an automatically sanitizable gap. In the daily work with monitoring data of thermal systems a maximum gap length of ten samples proved feasible. This will, however, still requires further analysis, depending on the expected results that the monitoring data shall yield. 


\subsection{Checking Data against Process Limits}

For selected data points it is reasonable to define process limits, such as e.g., a valid temperature range for inlet and outlet temperatures or maximum power consumption of an electric motor. When introducing knowledge about the process, a minimal configuration effort is introduced, requiring a level of information that is typically found on the data sheet of a component. When applying the data sanitation workflow it may be feasible to configure process limits for selected data points, given that the benefits outweigh the effort and that additional trust in the data can be gained. Three examples from real world experience shall illustrate this:

- Due to maintenance the configuration may change and thus the semantic link between controller and monitoring system may be broken, which can result in data rows being stored at wrong data points. Especially a local buffer that is periodically transferred to a central data collection is prone to such errors. Due to software updates the assignment between sensor identification and exported data rows may be reassigned, yielding to incorrect configuration.

- Electric and thermal meters often have preconfigured conversion factors that are used to convert the numeric meter value into a valid physical unit like $\mathrm{kWh}$. If this conversion factor is not properly set during commissioning or if it changes, e.g., due to replacement of the meter, the monitoring of historic values is broken.

- Unit conversions, e.g., from Kelvin to Celsius, also break the semantic link between numeric value and physical parameter, if they are not properly regarded.

All of the above issues can be identified by introducing process limits: incorrectly assigned data points will violate the upper or lower limit; for energy meters a maximum energy per period can be set-and checked by deriving the power from the recorded energy consumption; unit conversions are also detected, since the numeric values will likely not fit in the process limit. Section 5.5 shows an application of process limits by means of histograms as this is a convenient method to visualize the plausibility of data by separating valid from invalid data.

\subsection{Analysis of Data}

Under the assumption that data is free of outliers and is within process limits, the data quality can be further increased by introducing additional aspects of the process. Energy components in HVAC systems often have a clear system boundary that can be used to analyze the energy balance, mass balance or-derived from these balances-the power balance or mass flow balance. Energy balance can be examined when the main parameters are measured and stored-for example heat meters in the high temperature circuit, low temperature circuit and cooling tower circuit (as shown in the demonstration system in in Section 5.1). Data can then be validated based on the assumption that the energy in a system remains constant, resulting in the sum of all thermal energy flows to be zero. While this will not hold for strict mathematical equality, especially because thermal losses are not measured and thermal capacities differ, it still gives a good indicator on the plausibility of the data. In a similar way the data quality can be improved by analyzing the mass flows in a system to indicate possible issues in sensory equipment, calibration, commissioning or data transfer.

\section{Architectural Aspects of the Data Storage and Analysis System}

The process described in the previous section requires a set of methods and data views that are summarized in Table 1. It also requires a data storage system that has certain properties; the properties which are necessary for the scope of this paper are described in this section.

\subsection{Database System}

As described previously, monitoring of operation data in the field likely contains some incorrect values or values outside the specified range of operation. In the first steps of data collection all data 
has to be treated with caution, since it is not clear which data is trustable and which is not. Therefore raw data is never deleted or overwritten since the sanitation and correction algorithms may be rerun with improved configuration parameters. To achieve this, a layer model for data storage is used in this work (Figure 2). The data row Raw is populated during the import of a batch of raw data together with a timestamp of the sampling time (for illustration purposes, only hour and minute are shown in Figure 2). In this example a sample is taken every five minutes. Each sample has a tag faulty $(F)$ that is stored in the database. The outlier removal (Section 3.3), the check against process limits (Section 3.5) and the analysis by a domain expert (Section 3.6) may mark data samples as faulty $(F)$ indicating that these samples are not reliable. In the example in Figure 2, values between 0 and 60 are valid, resulting in the samples at 20:00 and 30:00 being marked as faulty. The figure also shows that there are no samples available at 00:05, 35:00 and 40:00.

\begin{tabular}{|c|c|c|c|c|c|c|c|c|c|c|c|c|}
\hline Timestamp[mm:ss] & $00: 00$ & & $10: 00$ & $15: 00$ & $20: 00$ & $25: 00$ & $30: 00$ & & & $45: 00$ & $50: 00$ & $55: 00$ \\
\hline \hline RAW & 20 & & 22 & 23 & 87 & 24 & -24 & & & 40 & 43 & 50 \\
\hline \hline $\mathrm{F}$ & 0 & & 0 & 0 & 1 & 0 & 1 & & & 0 & 0 & 0 \\
\hline
\end{tabular}

Figure 2. Raw data with faulty and missing data.

Data sanitation as described in Section 3.4 or for lager gaps in Section 5.6.1, operates on missing and faulty data and sanitizes the data row in order to get a consistent and plausible time series. The results are stored in a new data row in the database as shown in Figure 3. For sanitized samples the flag synthetic $(S)$ is set and a new data row Clean is filled with raw and synthetic data. The missing or faulty data are filled using linear interpolation; note that the Clean data row contains more samples than the Raw data row, since the missing samples at 00:05, 35:00 and 40:00 have been inserted.

\begin{tabular}{|c|c|c|c|c|c|c|c|c|c|c|c|c|}
\hline Timestamp [mm:ss] & $00: 00$ & $00: 05$ & $10: 00$ & $15: 00$ & $20: 00$ & $25: 00$ & $30: 00$ & $35: 00$ & $40: 00$ & $45: 00$ & $50: 00$ & $55: 00$ \\
\hline \hline Raw & 20 & & 22 & 23 & 87 & 24 & -24 & & & 40 & 43 & 50 \\
\hline \hline $\mathrm{F}$ & 0 & & 0 & 0 & 1 & 0 & 1 & & & 0 & 0 & 0 \\
\hline \hline $\mathrm{S}$ & 0 & 1 & 0 & 0 & 1 & 0 & 1 & 1 & 1 & 0 & 0 & 0 \\
\hline \hline Clean & 20 & 21 & 22 & 23 & 23,5 & 24 & 28 & 32 & 36 & 40 & 43 & 50 \\
\hline
\end{tabular}

Figure 3. The Clean data row containing raw and synthetic data after the sanitation process.

\subsection{Data Views}

Stored data can be accessed in different views. Many legacy systems can operate with data rows only on the base of time stamp and value. In case it is necessary to examine raw data with such a system, the additional meta-information on faulty and missing data cannot be transported. Therefore a view as shown in Figure 4 is used: all missing and faulty data are filled with zeroes, allowing the legacy system to operate with equidistant values free of gaps. Note that the Legacy data row is only a view that is not stored in the database, but is created upon request.

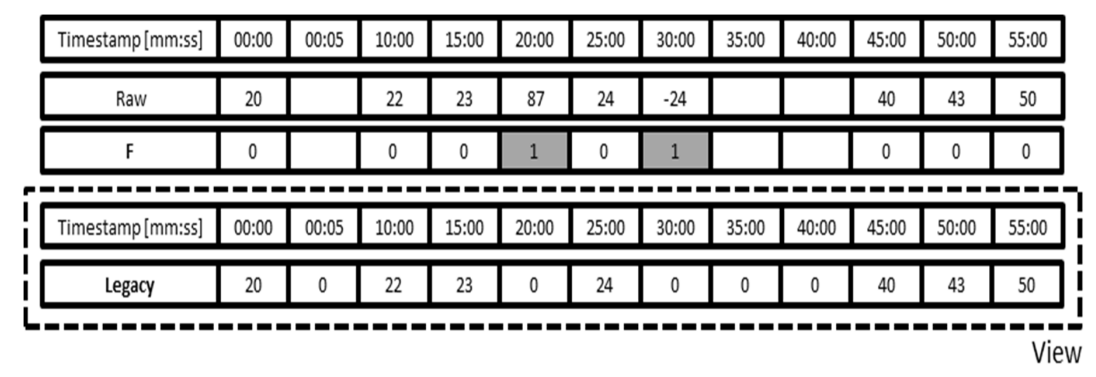

Figure 4. A view on the data for legacy system setting all faulty and missing data to zero. 
Another important method of supporting the user is to provide aligned data series. The data samples may be not be exactly equidistant, but rather may have jitter around the intended sample interval. In the example above, the time stamps may vary a few seconds as different sensors may be sampled too early or too late. For small jitter the Snap-to-grid view can be used. This view returns time stamps that are exactly equidistant and uses the closest sample to this timestamp. The underlying algorithm is executed only upon request and does not store the data in the database. It looks for a data sample within the sample interval and modifies its time stamp. Assuming that the sample interval has a sufficient resolution, the sample can just be taken as is. In case the sample interval changes (like in the following Sample-Interval-Variation view) the sample has to be interpolated between the neighboring samples in order to keep the error low.

Once a database is used that allows to separate stored data from viewed data, it is possible to query data with a sample interval different from the raw data sample interval. This becomes handy when e.g., temperatures with 5-min intervals and meter readings with 15-min intervals shall be compared in the same graph. The Sample-Interval-Variation view can deliver data with a specified sample interval, which may be shorter than the raw data interval in which case additional samples are interpolated or longer, meaning that several samples are averaged. Again the algorithm for varying the sample interval is executed on demand.

\subsection{Overview of Methods and Views}

The workflow shown in Figure 1 depends on a combination of methods and views that have to support human users who examine the data. They also need to be automatable in order to integrate periodic batches of newly imported data into the existing database. Table 1 lists the sequence of methods and views as they can be applied to raw data.

\section{Demonstration System: Adsorption Chiller}

This section applies the data sanitation workflow to an energy system that provides integrated monitoring of its main operation parameters. By analyzing the available data with regard to data quality, the workflow is validated and proven to be feasible. The execution of the workflow was set up such that the data related issues such as data access and outlier detection were addressed by IT experts. The validation steps that require limited domain knowledge were also done by IT experts with minor feedback from energy domain experts. After the workflow was executed the data was presented to energy experts and their feedback was provided: the data quality has been significantly increased, allowing discussions on how to further improve the design and the operation of the system. As a result the controller software has been updated to improve operation.

\subsection{System Setup}

The data used for this research has been taken from a water/silica gel based adsorption chiller, which is manufactured by the company Pink (Langenwang, Styria, Austria). The system uses hot water that is produced by solar power to produce cold water. Applications are, for example, cooling of meeting rooms and big event facilities, but also cooling facilities for food products. The design of the system is shown in Figure 5, showing the Low Temperature (LT), Medium Temperature (MT) and High Temperature (HT) cycles along with the installed sensors that are used for analysis. For the scope of this paper a total of 15 data points are used (Table 2).

The measured monitoring data is generated by the embedded controller of the chiller systems; it can be accessed via HTML requests using the built-in web server, which issues an XML document containing the current data. The data from the controller is recorded and stored in a monitoring and analysis system called OpenJEVis [27]. OpenJEVis is an open source system for monitoring and analyzing energy data, which provides data storage and visualization features. It also provides the meta-data structures for tagging data as faulty and allows for different views on the data. For accessing the data of the demonstration system, the flexible import features were used: a driver was 
written in Java to periodically poll the chiller controller. During storing, the data is not processed or validated; this is done later within the OpenJEVis system.

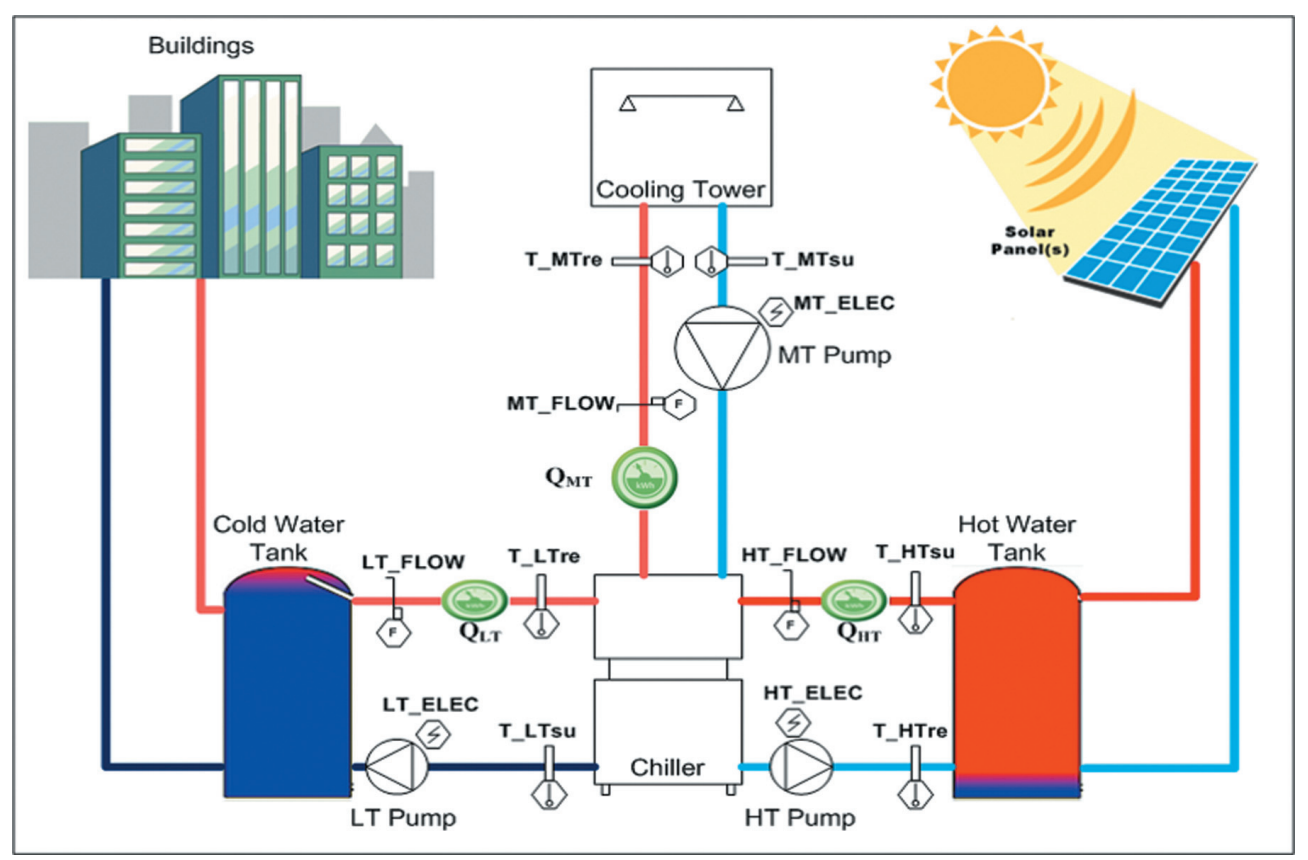

Figure 5. Adsorption chiller system configuration.

Table 2. Parameters Description.

\begin{tabular}{cc}
\hline Sensors & Description \\
\hline HT_Elec & Electricity consumption meter reading at high temperature cycle \\
MT_Elec & Electricity consumption meter reading at medium temperature cycle \\
LT_Elec & Electricity consumption meter reading at low temperature cycle \\
HT_Flow & Flow of water readings in high temperature cycle \\
MT_Flow & Flow of water readings in medium temperature cycle \\
LT_Flow & Flow of water readings in low temperature cycle \\
T_HTre & Temperature reading at high temperature cycle on return side \\
T_HTsu & Temperature reading at high temperature cycle on supply side \\
T_MTre & Temperature reading at medium temperature cycle on return side \\
T_MTsu & Temperature reading at medium temperature cycle on supply side \\
T_LTre & Temperature reading at low temperature cycle on return side \\
T_LTsu & Temperature reading at low temperature cycle on supply side \\
Q & Energy consumption reading at high temperature cycle \\
Q $_{\text {MT }}$ & Energy consumption reading at medium temperature cycle \\
Q & Energy consumption reading at low temperature cycle \\
\hline
\end{tabular}

\subsection{Data Availability of Monitoring Data}

Availability of monitoring data can be retrieved from the database by finding the gaps in data recording. In order to give a comprehensive overview of the availability a calendar view was chosen as shown in Figure 6. It shows the data availability of one chiller system, consisting of 15 data points (the parameters listed in Table 2) from June 2009 to October 2010 with a sampling interval of 4 min. Data is considered being missing if there is no data sample within a 4 min interval. The available data samples are counted and a color is assigned depending on the ratio of actual versus available data samples (in this example at most 15 data samples). 
The calendar view has different application in the data sanitation process: it may also be configured to show the availability of valid data, i.e., data that are not marked as faulty and are within process limits.

The calendar view does not require domain knowledge and provides a good overview. It is a starting point for further investigations, since the problematic time periods can be identified easily. In the given example the chiller is only in operation during the cooling season in summer, thus there is little data expected except for summer. The data gaps, e.g., on 6 September 2010, can be conveniently visualized.

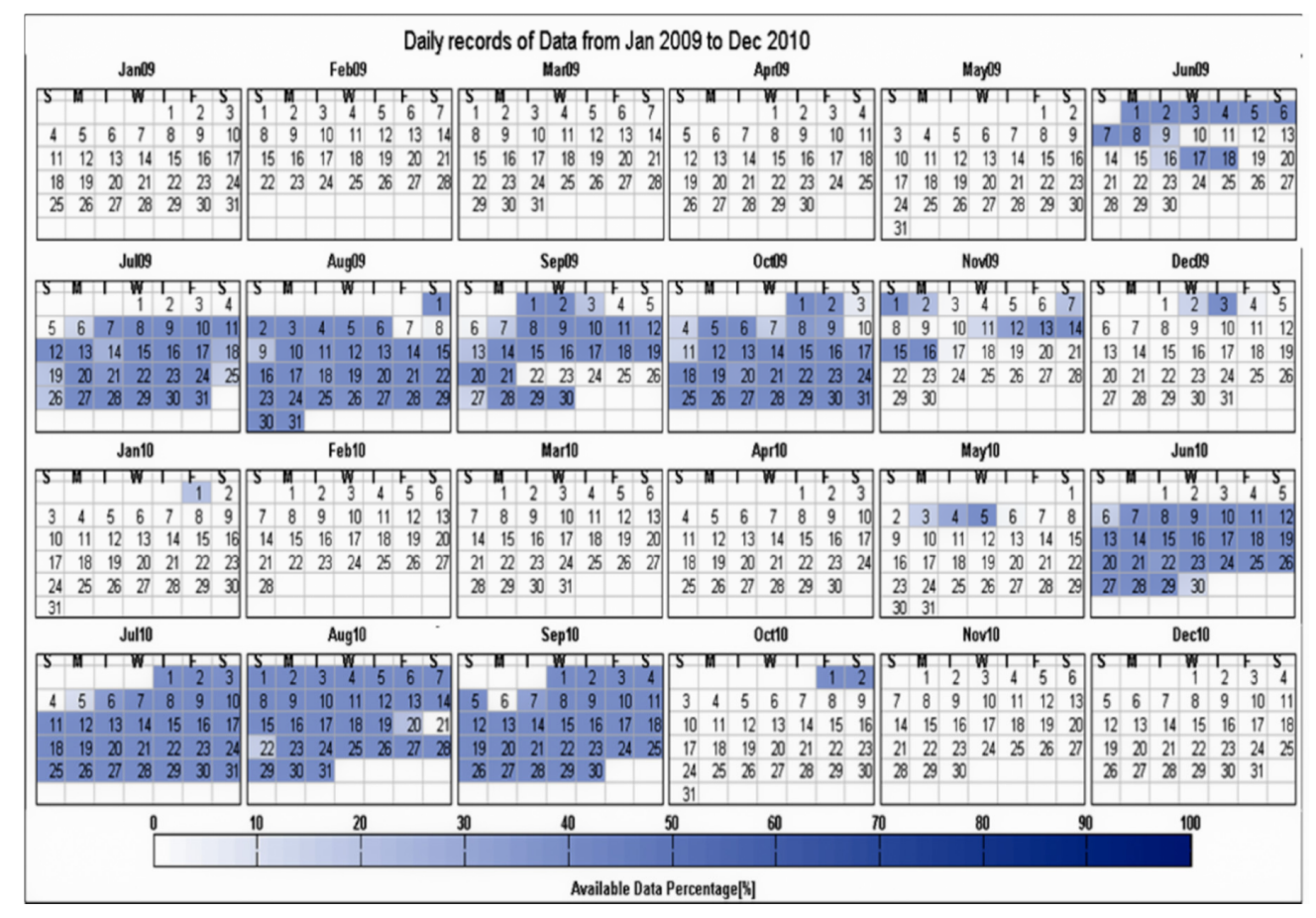

Figure 6. Calendar view of data availability: two years of operation, one system with 15 data points.

\subsection{On/Off State Detection and Duty Cycle Detection}

In order to detect the On/Off state and the resulting duty cycle automatically, the $k$-means clustering algorithm is used as explained in Section 3.2. Before using the $k$-means clustering, the data is normalized by using Min-Max normalization method with values in the range of 0 (minimum) to 1 (maximum). The number of clusters is set to two (On-state and Off-state) and the Euclidean distance is selected for finding the given number of clusters for both levels. The consecutive On states are considered as one cycle. The same process is repeated for all the consecutive On/Off states. The $k$-means algorithm is applied to monitoring data from the adsorption chiller in a period of 17 months (June 2009 to October 2010) and derives the state by using the 15 variables discussed in Table 2. The data is clustered automatically, meaning that after running the $k$-means algorithm the detected On/Off state can be stored and used as the base for the next steps of data analysis.

The detected On/Off states are shown in Figure 7 and demonstrate how the time series data are now supported by an additional On/Off state time series (the red line in the last graph of Figure 7 shows the On and Off-state). The dashed rectangle in Figure 7 shows that the rising of high and medium temperatures together with the decreasing low temperature verifies that the chiller is in operation and directly matches the detected On-state variable.

In order to validate the On/Off status by the $k$-means algorithm, the $k$-means algorithm has been applied to the five systems having a known On/Off status. Table 3 shows the accuracy rate of the $k$-means applied on the five systems. The average accuracy of the $k$-means is around $99 \%$. The 
$k$-means algorithm has lower accuracy rate in transient phase of the machine where the machines are just starting.

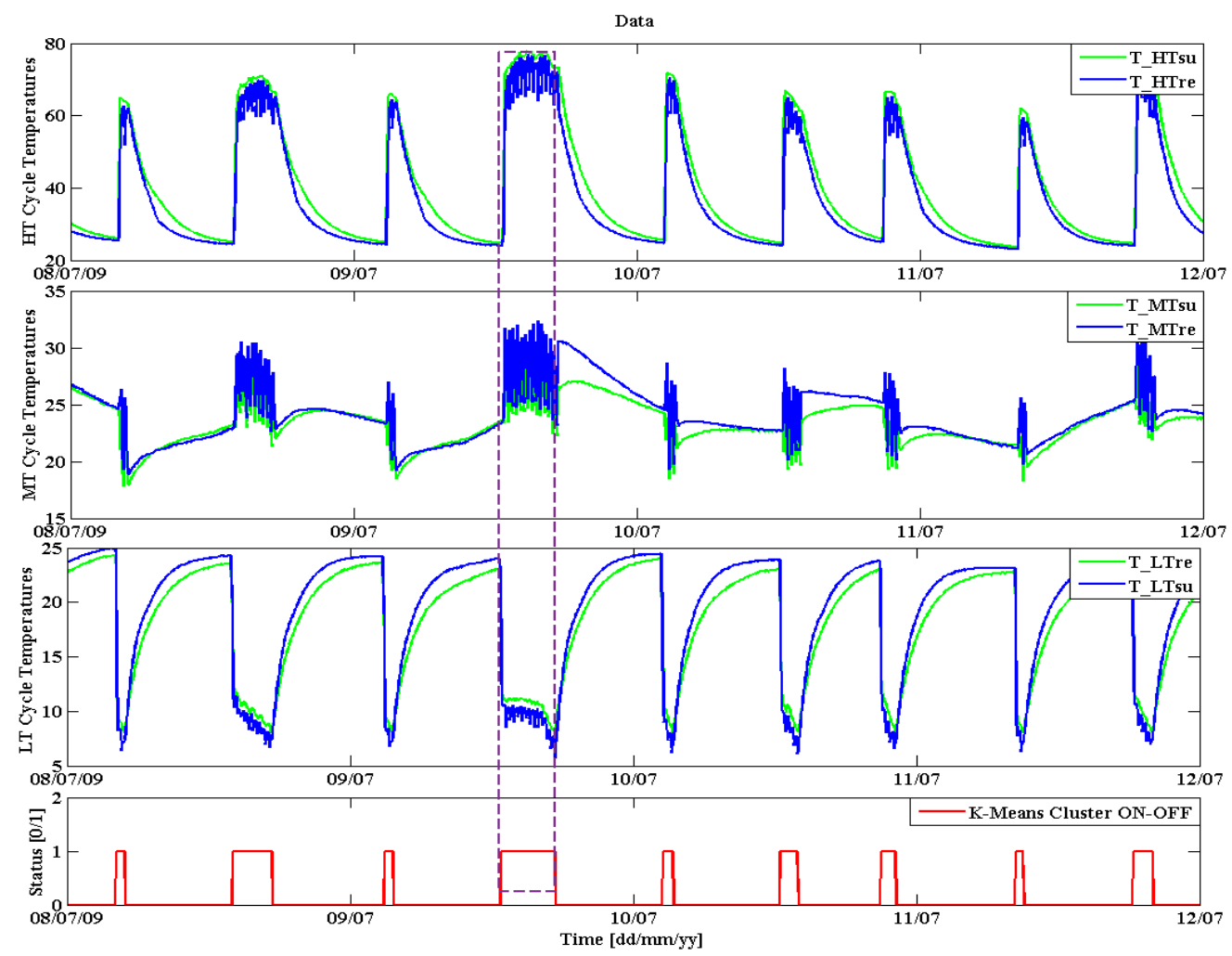

Figure 7. Automatically detected On/Off state time series using $k$-means clustering algorithm.

Table 3. K-Means On/Off accuracy.

\begin{tabular}{ccccc}
\hline S. No & System Name & From & To & $\begin{array}{c}\text { K-Means On/Off } \\
\text { Accuracy (\%) }\end{array}$ \\
\hline 1 & AEE-Gleisdorf (Machine 1) & 18 June 2013 & 9 March 2014 & $99.94 \%$ \\
2 & Behmann-Egg (Machine 2) & 25 June 2013 & 28 April 2015 & $99.42 \%$ \\
3 & HIZ-Zeltweg (Machine 3) & 24 July 2013 & 27 April 2015 & $95.77 \%$ \\
4 & Koegerlhof-Hartmannsdorf (Machine 4) & 27 Janurary 2014 & 27 April 2015 & $99.92 \%$ \\
5 & Privathaus-Niederoesterreich (Machine 5) & 10 March 2013 & 27 April 2015 & $99.21 \%$ \\
\hline
\end{tabular}

\subsection{Outlier Detection Using Z-Score Normalization}

In order to detect outliers in the data the duty cycle based z-score is applied as described in Section 3.3. The statistical data for the z-scores ( $\mu$ and $\sigma$ ) are derived based on single duty cycles instead of e.g., a whole day. During the transient phase where the chiller is changing its state from either On to Off or vice versa, the initial behavior does not exhibit the normal behavior of the cycle. Therefore the transient phase of $30 \mathrm{~min}$ during Off-state and $4 \mathrm{~min}$ of On-state are not considered for the mean and standard deviation of the respective cycle. Figure 8 shows the original data (T_LTre) along with the duty cycle z-score; the detected outliers using expectation maximization clustering (EM) algorithm are marked with red circles both in the original data and in the z-scored data. These outliers are, however, only single samples and can be restored by simple gap filling as described in Section 5.6.1. Furthermore, the change of specific On-state and Off-state z-score parameters allows for better detection of outliers, since the behavior of a process can strongly differ between two operational states. 


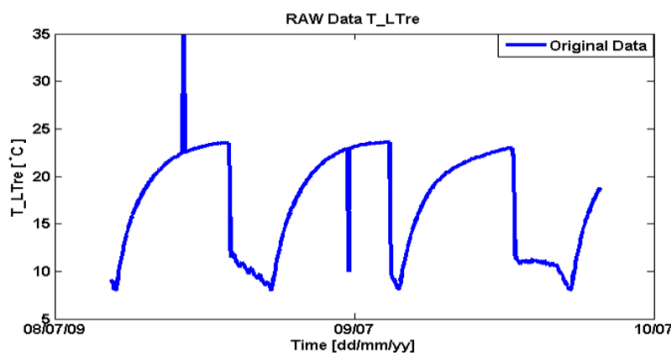

(a)

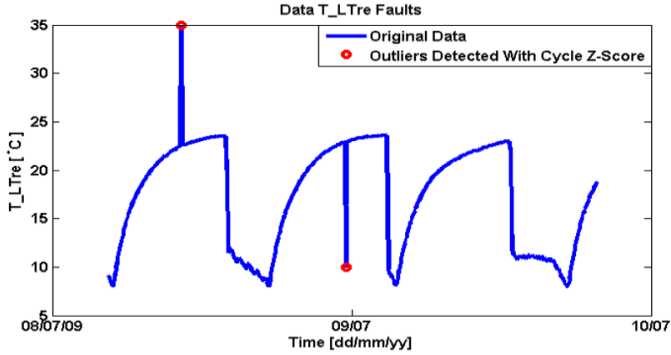

(c)

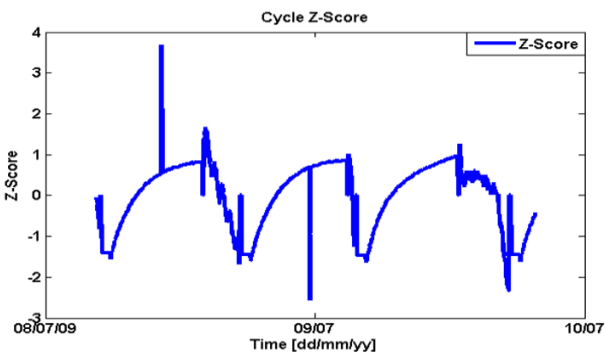

(b)

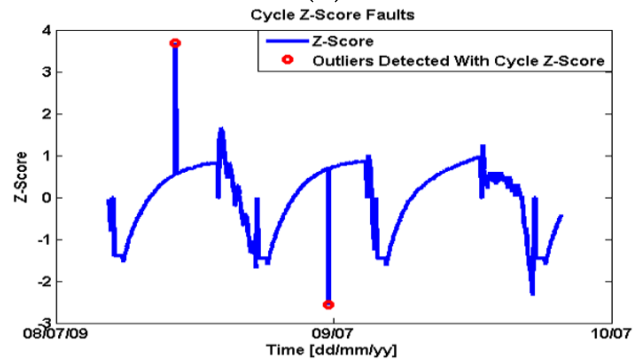

(d)

Figure 8. Outlier detection using z-scores: (a) Raw data of T_LTre; (b) cycle based z-score representation of the raw data; (c) faults detected in the raw data; (d) faults detected in the corresponding z-score representation.

In order to compare the cycle based z-score outlier detection with normal z-score based outlier detection, the data has been tested with both methods. Figure 9 represents the results of the tests done with normal z-score and cycle based z-score. It can be observed that cycle based z-score outlier detection has detected faults in data that were away from normal behavior in a cycle such as with the outlier detected close to 9 July 2009. In contrast, the normal z-score outlier detection method only detected those points that were far away from the behavior of the whole data.

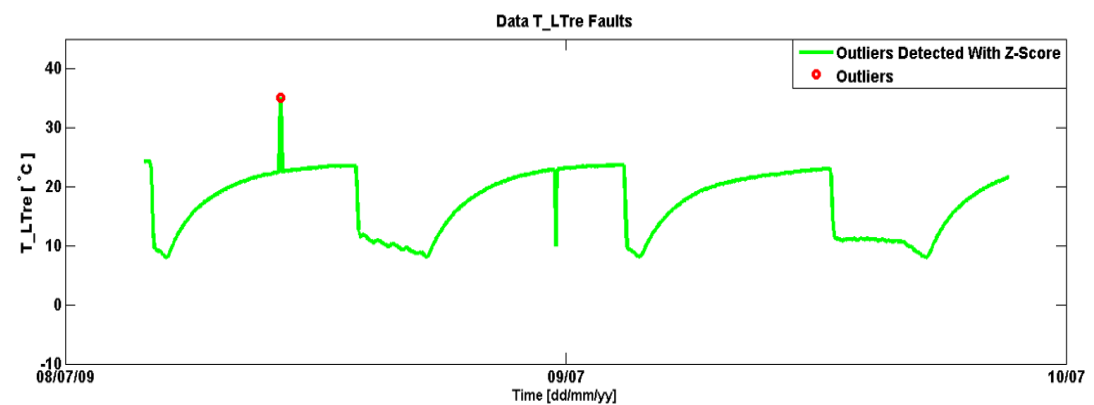

(a)

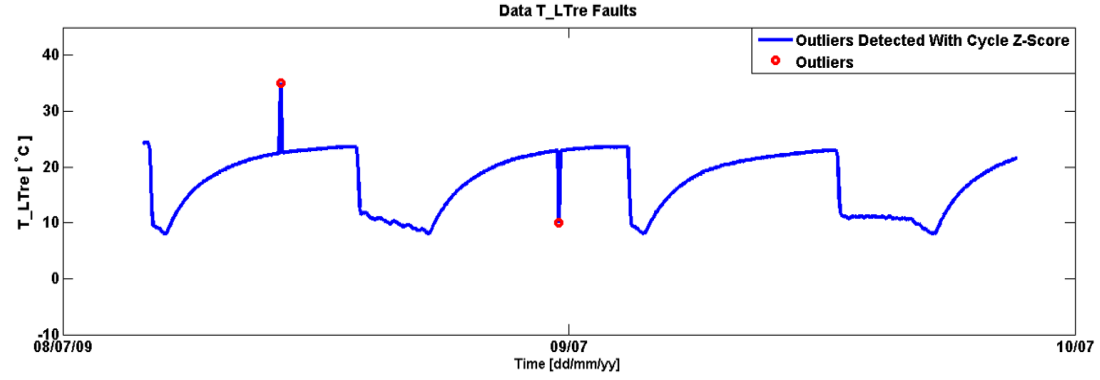

(b)

Figure 9. Outliers in data (a) Outliers detected with normal z-score (b) Outliers detected with cycle based z-score. 


\subsection{Visualization of Process Limits}

The check against process limits as described in Section 3.5 can be used to visualize statistical information about the data distribution. In combination with duty cycle detection this provides a swift way of visually checking data plausibility: by visualizing only data from the On-state of the system, the number of process limit violations is strongly reduced, thus giving insight into the real operation of the system. If data from the Off-cycle was also regarded, there would be a large amount of (irrelevant) violations. Figures 10 and 11 show two system temperatures in specifically adapted histograms: the data within the process limits are equally divided into ten bins; on the upper and lower boundary there is one additional process limits violation bin that contains all remaining outliers (for better visualization these two bins are drawn with red bars, see Figure 10). Figure 11 contains data with no outliers, which is indicated by the violation bins to have $+\infty$ as the upper boundary and $-\infty$ as the lower boundary. This allows to check that there is not a single violation in this data.

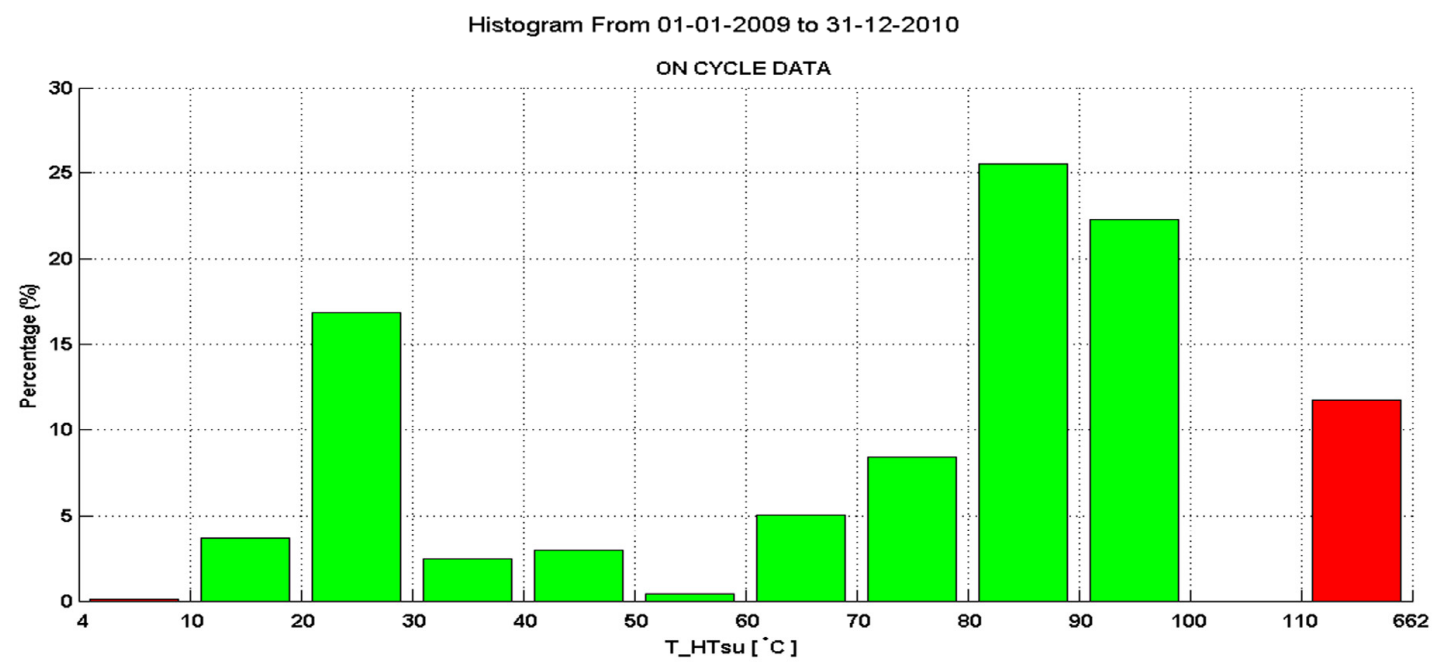

Figure 10. Histogram of the supply temperature in the high temperature cycle showing only the temperatures while the system is on. The data contains outliers.

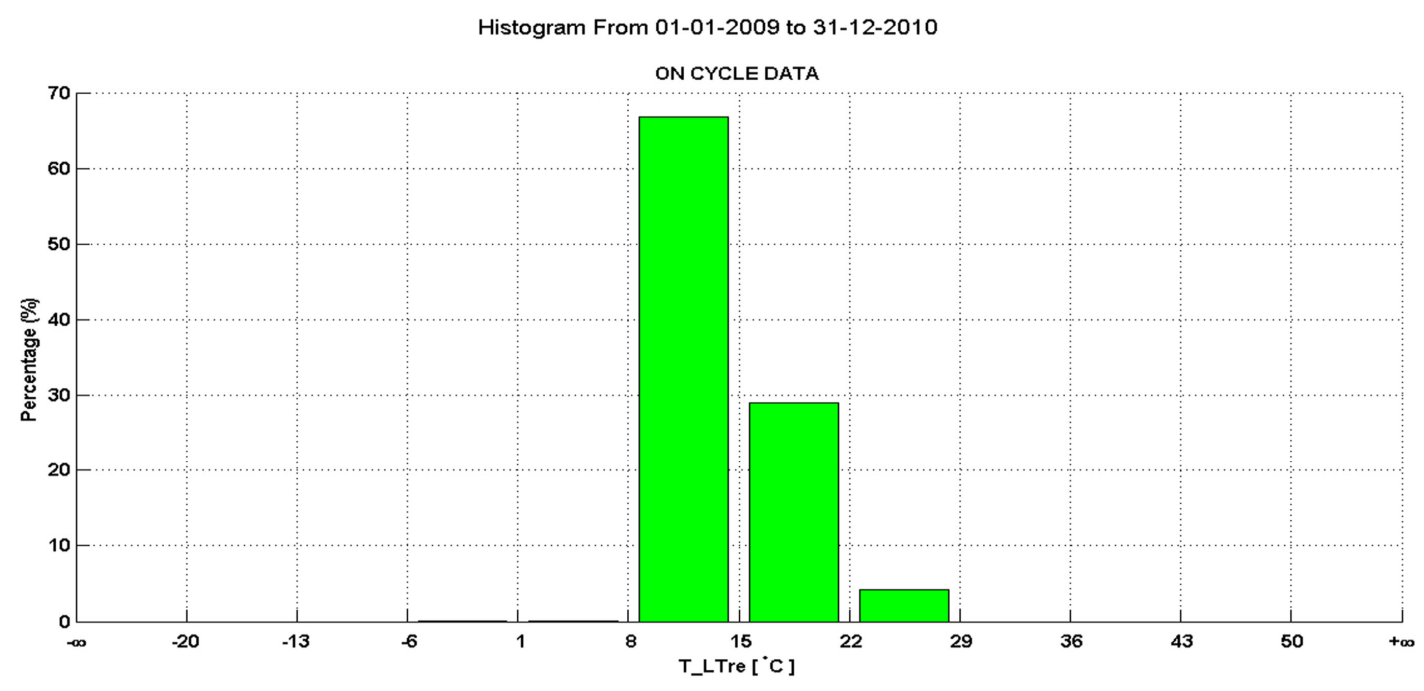

Figure 11. Histogram of the return temperature in the low temperature cycle showing only the temperatures while the system is on, showing no outliers (violation bins on the left and right are empty). 


\subsection{Analysis}

Based on the previous sanitation steps the data is prepared for high-level and domain-specific analysis. This section covers three methods: at first the issue of large gaps is addressed, because this is a common situation found when recording monitoring data. Then the data is analyzed using process knowledge about the energy flows in the system; finally an important performance indicator (Coefficient of Performance, COP) for thermal energy systems is derived using the sanitized data.

\subsubsection{Synthetic Profiles for Large Gaps}

In the case where there are long missing gaps in the data the regression by discretization [28] method has shown better results as compared to linear interpolation. The process of regression by discretization consists of the following steps:

(1) Data is divided into a set of intervals. The length of each interval heavily affects the regression results.

(2) Then each interval is assigned a discrete class value based on mean, mode or some other measure [29]. Any output that will have a similar kind of interval will be assigned the same class value.

Figure 12 shows original data on the top left and an artificial data gap that is introduced on the top right. While linear interpolation creates a time graph as shown on the lower right, the regression model being used reconstructs the missing data as shown on the lower left side.
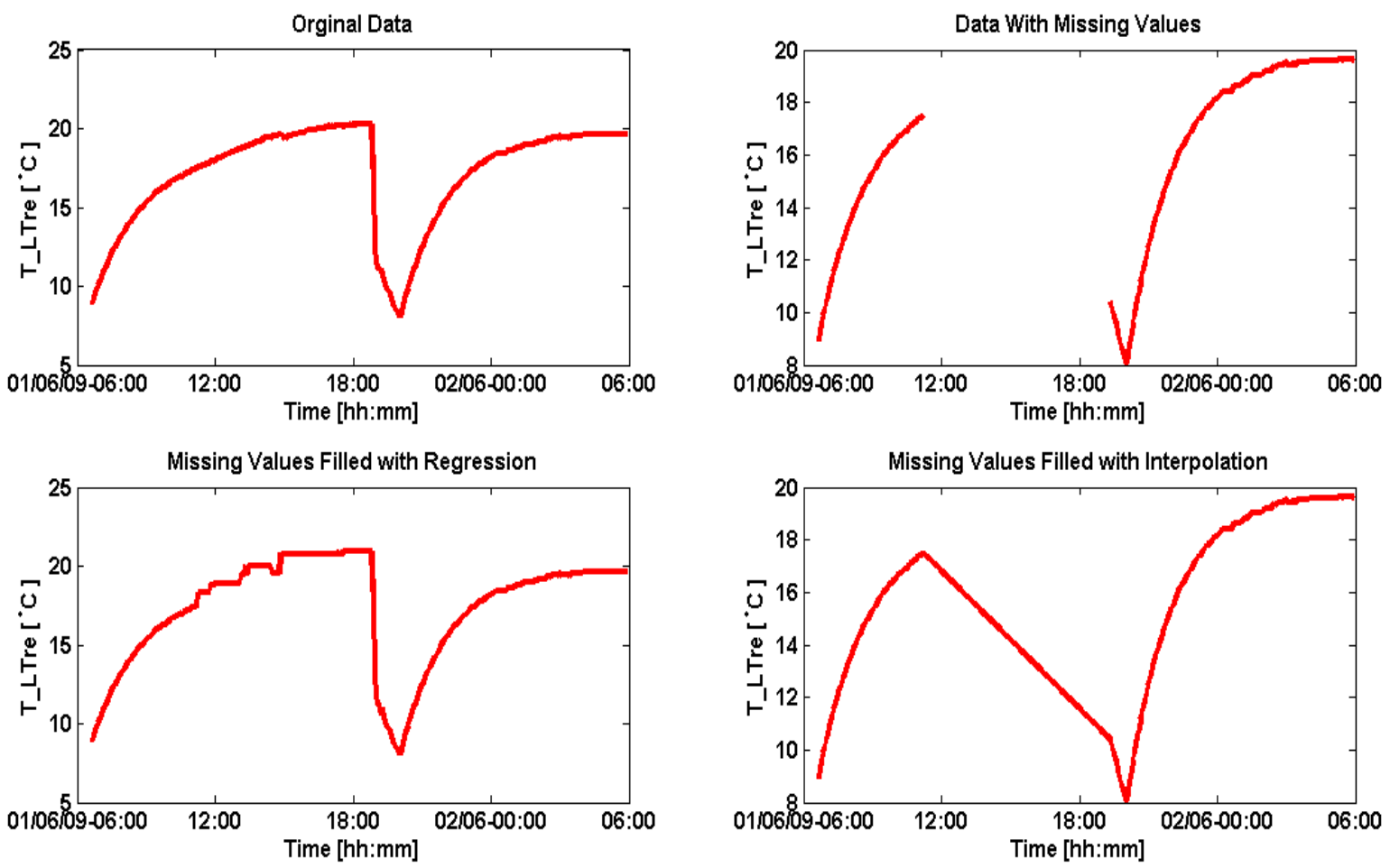

Figure 12. Sanitation of large gaps using regression.

\subsubsection{First Principle Analysis: Energy Flux Balance}

The first law of thermodynamics can be used as first principles for detection of faults in the data, given that the system boundaries are known and all relevant parameters are measured. The demonstration system has three heat meters measuring the heat flux in the low, medium and high temperature cycle. This allows to define a system boundary where only the losses are unknown. It is 
required that the energy that flows into the system should be equal to the energy that flows out of the system or is stored in the system:

$$
Q_{\mathrm{LT}}+Q_{\mathrm{HT}}-Q_{\mathrm{MT}}+\Delta E=0
$$

Therefore:

$$
\Delta E=-Q_{\mathrm{LT}} \pm Q_{\mathrm{HT}} \pm Q_{\mathrm{MT}}
$$

Here $\Delta E$ represents the combination of losses and changes in the stored energy. Since the chiller does not have a significant thermal capacity, the assumption to verify is that $\Delta E$ should be close to 0 . Figure 13 shows $\Delta E$ for eight duty cycles of the adsorption chiller. The values have high dynamics when deriving the energy balance on a sample-by-sample base (blue graph). This is expectable due to the various delays in thermal dissipation and mass flows. When looking at the average over a duty cycle (red graph) the energy balance ranges from $-0.2 \mathrm{~kW}$ to $0.2 \mathrm{~kW}$, which is an acceptable range. In conclusion, the measured data is therefore resilient, the energy balance is plausible and the metering data can be used for further analysis. The automated detection of the duty cycle contributes to this analysis, since the data in Off-state are commonly not relevant for energy balance analysis.

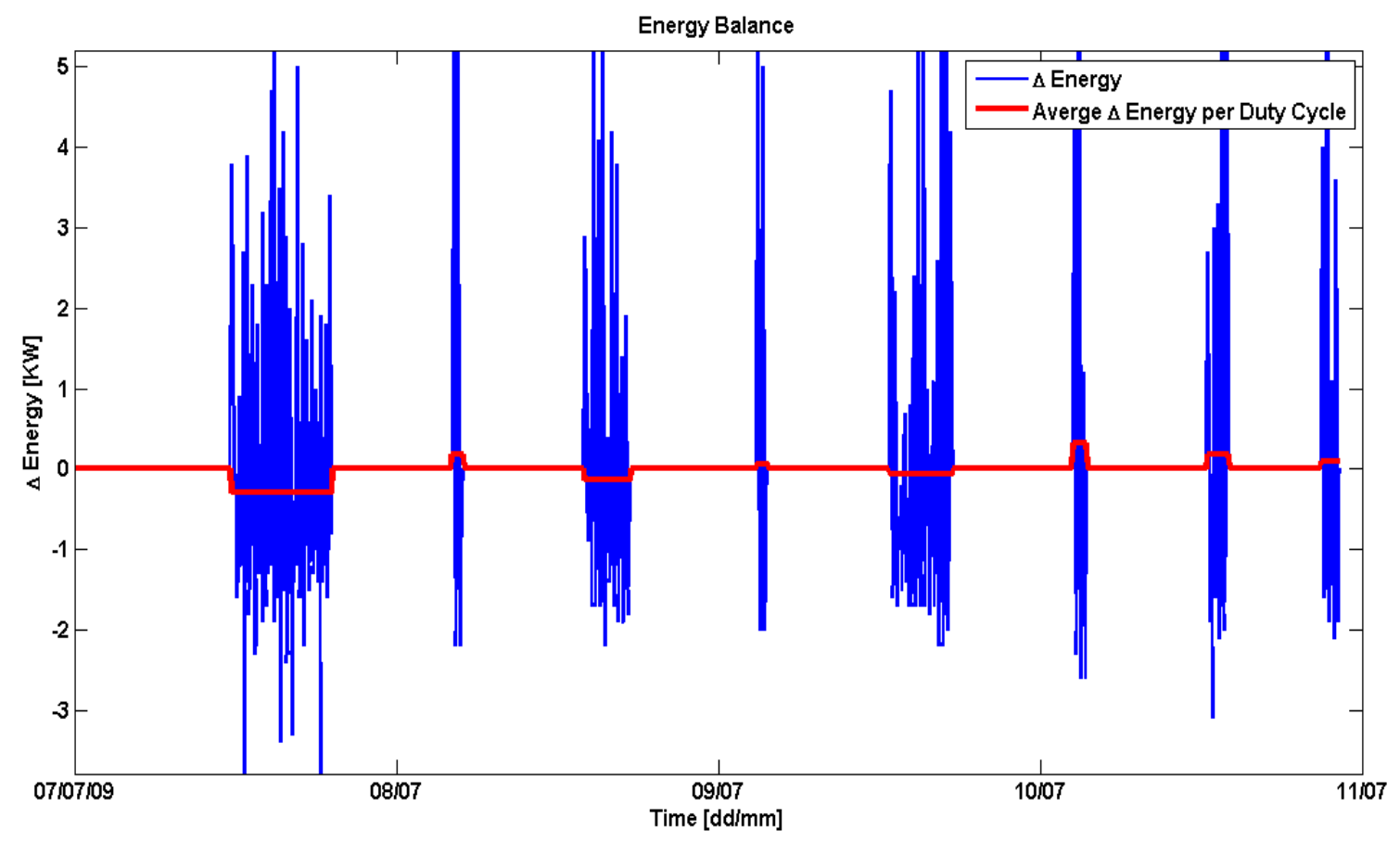

Figure 13. Energy flux balance in an adsorption chiller.

\subsubsection{Thermal Coefficient of Performance ( $\left.C O P_{\text {therm }}\right)$ Calculation}

The thermal coefficient of performance $\left(C O P_{\text {therm }}\right)$ is another analysis method to improve data quality and to identify faults. The $C O P_{\text {therm }}$ of an adsorption chiller has to be in a range between 0 and 1 , a $C O P_{\text {therm }}$ outside this range is physically not possible in steady state and therefore is an indicator for low data quality. The $C O P_{\text {therm }}$ is derived using:

$$
C O P_{\text {therm }}=\frac{Q_{\mathrm{LT}}}{Q_{\mathrm{HT}}}
$$

Figure 14 shows the thermal COP derived on a sample-by-sample base (blue graph) and averaged over the On-state of a duty cycle (red graph). Similar to the energy flux balance in the previous section the average value gives a good indication that the data is plausible. 
Figure 15 shows the average $C O P$ of cycles for one cooling season. The lower $C O P$ ranging from 0 to 0.1 are cycles with short operational times. The average COP shows that for most cycles the system performed in the range from 0.3 to 0.6 , indicating good performance.

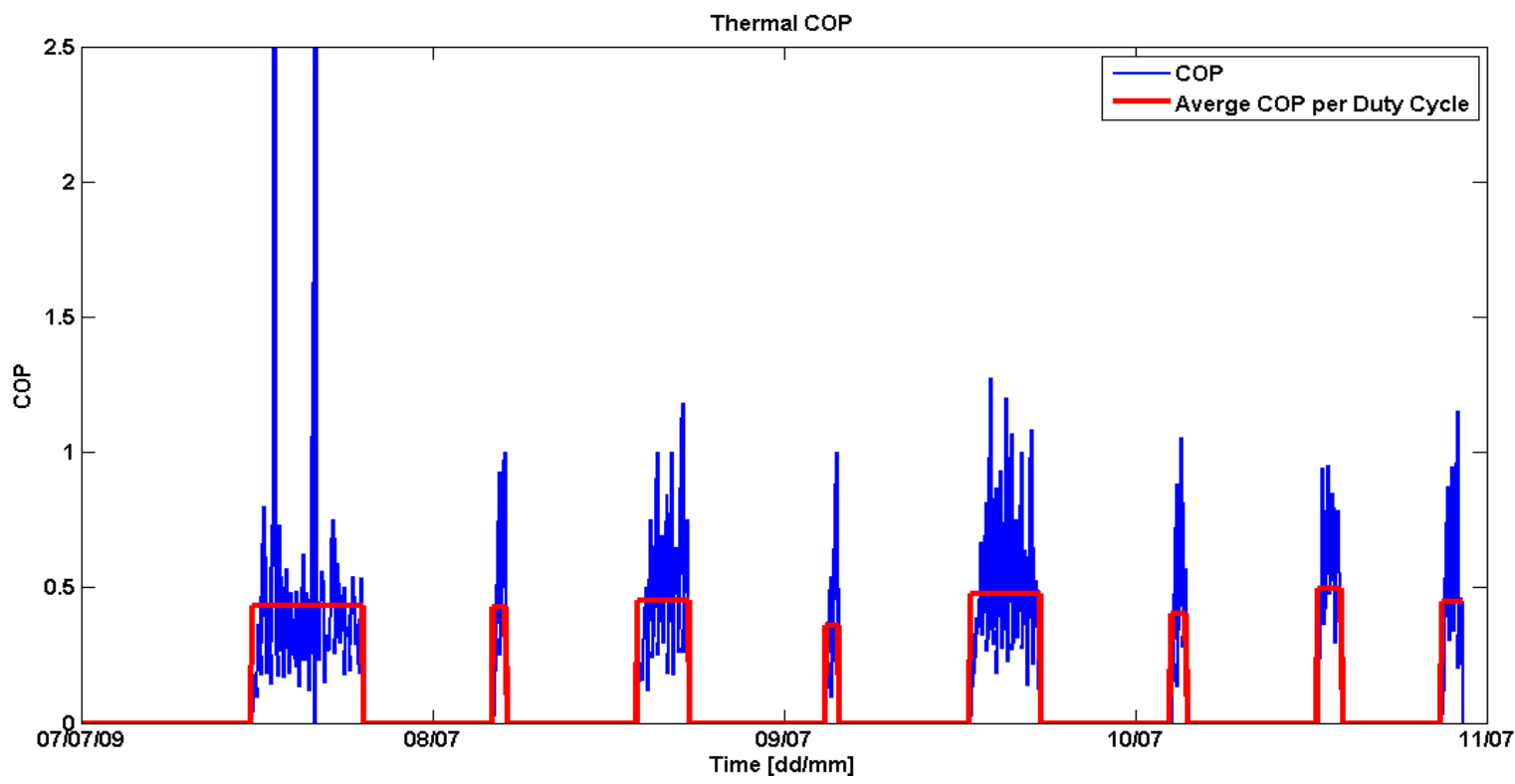

Figure 14. Coefficient of Performance (COP) on a sample-by-sample base and in average over the On-state of a duty cycle.

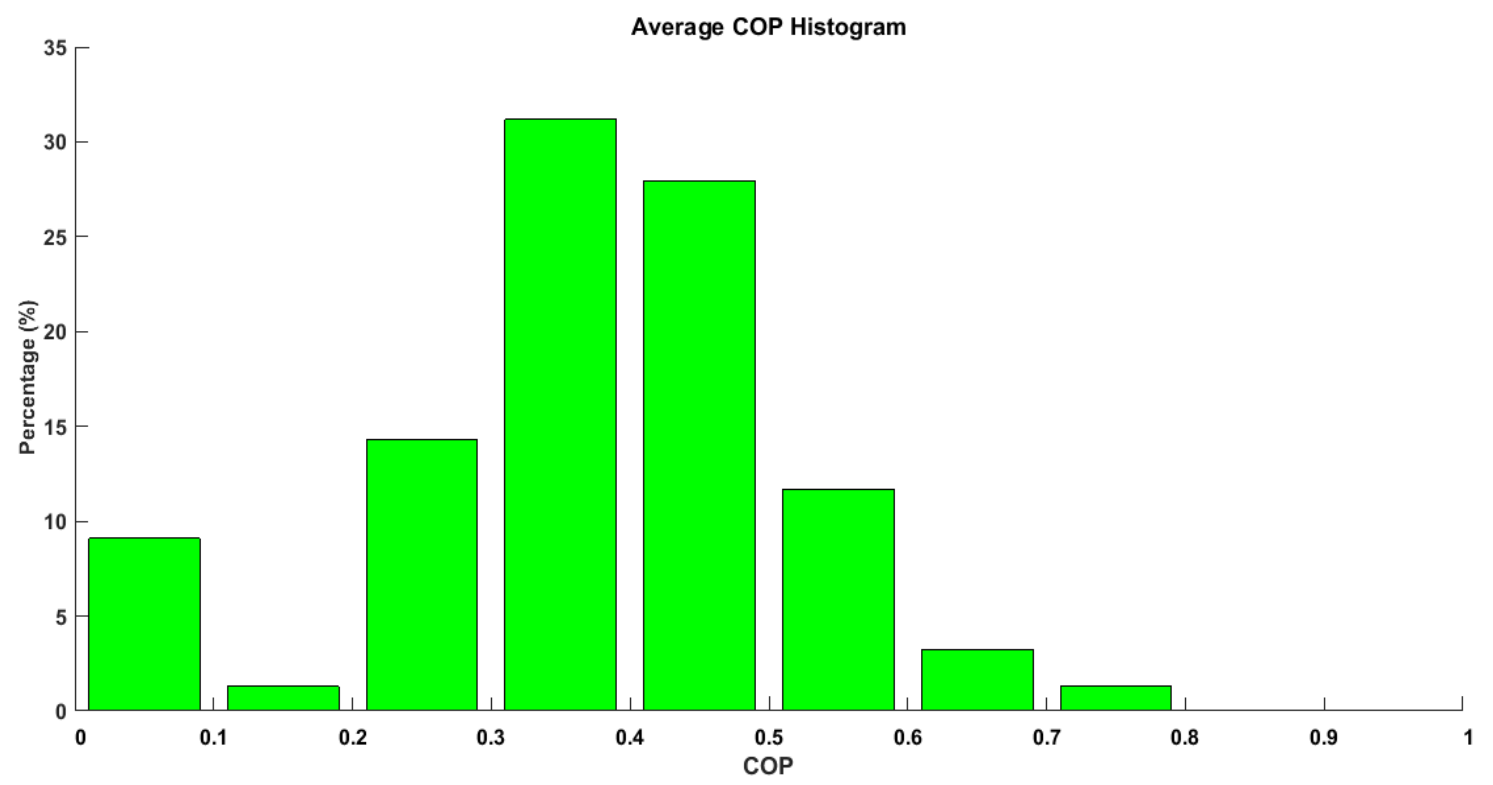

Figure 15. Average COP per cycle histogram.

\section{Conclusions and Outlook}

The workflow presented in this paper is designed towards minimal configuration effort in order to allow efficient data analysis. Also the separation between an Information Technology (IT) operator who does the sanitation of data and the domain expert who analyzes the sanitized data, strongly contributes to focusing resources on the actual analysis work. The workflow has been successfully applied to verify real monitoring data of absorption chillers. It was shown that the data quality can be significantly improved, thus leaving more time for domain-specific analysis. The combination of 
automated duty cycle detection, clustering, outlier detection algorithms and gap sanitation allows to quickly increase the data quality and provides a solid base for following analyses. Furthermore, examples of analysis are shown using the derived energy balance and automated COP calculation only in On-state. The workflow is clearly limited to time series data and has a strong focus towards duty cycle based data as they occur in the operation of energy systems and is not generally applicable to e.g., data mining scenarios. However, more work is required to make the workflow generally applicable to a broader portfolio of energy systems with even less configuration and commissioning effort. This will require semi-automated, semantic data analysis to grasp the meaning of data rows even when semantic information is missing or incomplete.

Future work will focus on enlarging the portfolio of available methods and refine the existing methods based on further experiences by domain experts. More analysis steps will be introduced, regarding, amongst others, the automated detection of stuck-at-value errors in the data (i.e., data that indicates that the according data point does not deliver valid data, but only repeats the last value infinitely) and the automated detection of inefficient periods of operation.

Acknowledgments: This work was partly funded by the Austrian Funding Agency in the funding programme e!MISSION within the project "extrACT", Project No. 838688.

Author Contributions: Gerhard Zucker, Usman Habib and Florian Judex conceived and designed the experiments; Usman Habib performed the experiments Gerhard Zucker, Usman Habib and Florian Judex analyzed the data; Usman Habib., Max Blöchle and Thomas Leber contributed IT and analysis tools Gerhard Zucker and Usman Habib wrote the paper.

Conflicts of Interest: The funding agency had no role in the design of the study: neither in the collection, analyses, or interpretation of data; in the writing of the manuscript, or in the decision to publish the results. The authors declare no conflict of interest.

\section{References}

1. XAMControl. Available online: http://www.evon-automation.com/index.php/en/products/xamcontrol (accessed on 13 October 2015).

2. Enterprise Building Integrator (EBI). Available online: https://www.ebi.honeywell.com/en-US/Pages/ homepage.aspx (accessed on 13 October 2015).

3. Desigo CC—Building Technologies—Siemens. Available online: http://www.buildingtechnologies.siemens. com/bt/global/en/building-solutions/desigo-cc/Pages/desigo-cc.aspx (accessed on 13 October 2015).

4. Mourad, M.; Bertrand-Krajewski, J.-L. A method for automatic validation of long time series of data in urban hydrology. Water Sci. Technol. 2002, 45, 263-270. [PubMed]

5. Handbook of Automated Data Quality Control Checks and Procedures; US Department of Commerce: Washington, DC, USA, 2009; p. 78.

6. Sun, S.; Bertrand-Krajewski, J.-L.; Lynggaard-Jensen, A.; Broeke, J.V.D.; Edthofer, F.; Almeida, M.D.C.; Ribeiro, À.S.; Menaia, J. Literature Review for Data Validation Methods; Deliverable of Project PREPARED: Nieuwegein, Utrecht, The Netherlands, 2012.

7. Mattarelli, A.; Piva, S. EN 15316 Calculation Methods for the Generation Sub-System: The Influence of Input Data on the Results. Energy Proc. 2014, 45, 473-481. [CrossRef]

8. Pipino, L.L.; Lee, Y.W.; Wang, R.Y. Data quality assessment. Commun. ACM 2002, 45, 211-218. [CrossRef]

9. Branisavljević, N.; Kapelan, Z.; Prodanović, D. Improved real-time data anomaly detection using context classification. J. Hydroinformatics 2011, 13, 307-323. [CrossRef]

10. Olsson, G.; Nielsen, M.; Yuan, Z.; Lynggaard-Jensen, A.; Steyer, J.-P. Instrumentation, Control and Automation in Waste Water Systems; IWA Publishing: London, UK, 2005; p. 264.

11. Katipamula, S.; Brambley, M.R. Review Article: Methods for Fault Detection, Diagnostics, and Prognostics for Building Systems-A Review, Part I. HVACR Res. 2005, 11, 3-25. [CrossRef]

12. Fan, C.; Xiao, F.; Wang, S. Development of prediction models for next-day building energy consumption and peak power demand using data mining techniques. Appl. Energy 2014, 127. [CrossRef]

13. Fayyad, U.; Piatetsky-Shapiro, G.; Smyth, P. From data mining to knowledge discovery in databases. AI Mag. 1996, 17, 37. 
14. Wu, S.; Sun, J.-Q. Cross-level fault detection and diagnosis of building HVAC systems. Build. Environ. 2011, 46, 1558-1566. [CrossRef]

15. Schein, J.; Bushby, S.T.; Castro, N.S.; House, J.M. A rule-based fault detection method for air handling units. Energy Build. 2006, 38, 1485-1492. [CrossRef]

16. Yu, Z.J.; Haghighat, F.; Fung, B.C.; Zhou, L. A novel methodology for knowledge discovery through mining associations between building operational data. Energy Build. 2012, 47, 430-440. [CrossRef]

17. Zucker, G.; Malinao, J.; Habib, U.; Leber, T.; Preisler, A.; Judex, F. Improving energy efficiency of buildings using data mining technologies. In Proceedings of the 2014 IEEE 23rd International Symposium on Industrial Electronics (ISIE), Istanbul, Turkey, 1-4 June 2014; pp. 2664-2669.

18. Yu, F.W.; Chan, K.T. Improved energy management of chiller systems by multivariate and data envelopment analyses. Appl. Energy 2012, 92, 168-174. [CrossRef]

19. Hong, T.; Yang, L.; Hill, D.; Feng, W. Data and analytics to inform energy retrofit of high performance buildings. Appl. Energy 2014, 126, 90-106. [CrossRef]

20. Hartigan, J.A.; Wong, M.A. Algorithm AS 136: A k-means clustering algorithm. Appl. Stat. 1979, 28, 100-108. [CrossRef]

21. Lloyd, S. Least squares quantization in PCM. IEEE Trans. Inf. Theory 1982, 28, 129-137. [CrossRef]

22. Jain, A.K. Data clustering: 50 years beyond K-means. Pattern Recognit. Lett. 2010, 31, 651-666. [CrossRef]

23. Marx, M.L.; Larsen, R.J. Introduction to Mathematical Statistics and Its Applications; Pearson/Prentice Hall: Upper Saddle River, NJ, USA, 2006.

24. Jin, X.; Han, J. Encyclopedia of Machine Learning; Claude, S., Webb, G.I., Eds.; Springer US: New York, NY, USA, 2011; pp. 382-383.

25. Smyth, P. Model selection for probabilistic clustering using cross-validated likelihood. Stat. Comput. 2000, 10, 63-72. [CrossRef]

26. Smyth, P. Clustering Using Monte Carlo Cross-Validation. In Proceedings of the Second International Conference on Knowledge Discovery and Data Mining (KDD), Portland, OR, USA, 2-4 August 1996; pp. 126-133.

27. Palensky, P. The JEVis System-An Advanced Database for Energy-Related Services. Available online: http:/ / www.actapress.com/PaperInfo.aspx?PaperID=13022\& reason=500 (accessed on 3 March 2015).

28. Torgo, L.; Gama, J. Search-based Class Discretization. In Proceedings of the Ninth European Conference on Machine Learning, Prague, Czech Republic, 23-25 April 1997; pp. 266-273.

29. Frank, E.; Bouckaert, R.R. Conditional Density Estimation with Class Probability Estimators. In Advances in Machine Learning; Zhou, Z.-H., Washio, T., Eds.; Springer: Berlin, Germany, 2009; pp. 65-81.

(C) 2015 by the authors; licensee MDPI, Basel, Switzerland. This article is an open access article distributed under the terms and conditions of the Creative Commons by Attribution (CC-BY) license (http:/ / creativecommons.org/licenses/by/4.0/). 